\title{
Pathophysiology of Temporomandibular Joint Arthritis: Review
}

\author{
Hye-Min Ju', Kyung-Hee Kim², Sung-Hee Jeong ${ }^{3}$, Yong-Woo Ahn ${ }^{3}$, Soo-Min Ok ${ }^{3}$ \\ 'Department of Oral Medicine, Pusan National University Dental Hospital, Dental Research Institute, Yangsan, Korea \\ ${ }^{2}$ Department of Oral Medicine, Inje University Busan Paik Hospital, Busan, Korea \\ ${ }^{3}$ Department of Oral Medicine, Pusan National University, School of Dentistry, Dental Research Institute, \\ Dental and Life Science Institute, Yangsan, Korea
}

Received September 2, 2021

Revised September 17, 2021

Accepted September 17, 2021

\author{
Correspondence to: \\ Soo-Min Ok \\ Department of Oral Medicine, Pusan \\ National University, School of Dentistry, \\ Dental Research Institute, Dental and Life \\ Science Institute, 49 Busandaehak-ro, \\ Mulgeum-eup, Yangsan 50612, Korea \\ Tel: +82-55-360-5243 \\ Fax: +82-55-360-5238 \\ E-mail: oksoomin@pusan.ac.kr \\ https://orcid.org/0000-0003-1776-371X \\ This work was supported by a 2-year \\ Research Grant of Pusan National University.
}

As for temporomandibular joint arthritis (TMJ OA), managing the contributing factors at an early stage through accurate diagnosis is necessary to prevent irreversible bone changes. TMJ OA, which is a multi-organ disease caused by various pathophysiological mechanisms, is developed mainly due to mechanical overload. It is a disease characterized by degeneration of articular cartilage and subchondral bone as a low-level inflammatory arthritis condition developed by dysregulation of catabolic and anabolic activity of chondrocytes. Age, mechanical overload sensing of cartilage, chondrocyte apoptosis, catabolic enzymes, inflammatory factors, abnormal remodeling of subchondral bone, and estrogens may be involved in the pathogenesis of arthritis. Therefore, a comprehensive evaluation is needed to diagnose and manage progressive cartilage degeneration, subchondral bone remodeling, and associated symptoms of TMJ OA.

Key Words: Pathophysiology; Temporomandibular joint; Temporomandibular joint arthritis; Temporomandibular joint disorder

\section{INTRODUCTION}

Temporomandibular joint arthritis (TMJ OA) affects $8 \%-16 \%$ of the population, is more common in women, and increases with age [1]. TMJ OA is described as a degenerative joint disease in the Diagnostic Criteria for Temporomandibular disorders (DC/TMD). The TMJ OA shows osseous changes and degeneration of surrounding tissues in one or both of the temporomandibular joints. In addition, the patients complain of crepitation on history taking. However, osteoarthritis is a disease with low sensitivity and specificity compared to other diseases defined by DC/TMD. Computer tomography scans of TMJ OA show erosion, sclerosis, pseudocyst, and flattening of articular eminence and/or condyle [2].

TMJ OA is a multifactorial disease that is caused by various pathophysiological mechanisms [3]. The temporomandibular joint is a complex organ composed of several tissues such as the masticatory muscle ligament, articular disc, bone, and cartilage. These constituent tissues interact with heredity, environment, nerves and hormones, which in turn causes TMJ OA [3]. Therefore, a comprehensive evaluation is needed to diagnose and manage progressive cartilage degeneration, subchondral bone remodeling, and associated symptoms of TMJ OA [4]. To this end, the mechanism and associated factors of TMJ OA are reviewed to establish the background data necessary for the early and accurate diagnosis of TMJ OA. 


\section{THE STRUCTURE AND BIOMECHANICAL PROPERTIES OF THE TEMPOROMANDIBULAR JOINT}

The mandibular condylar cartilage is a multiple layered tissue that plays an important role in the function of the temporomandibular joint. Mandibular condylar cartilage reduces the load on the subchondral bone with the joint disc, and is composed of fibrocartilage unlike most of other joints. The fibrous layer of mandibular condylar cartilage is on its surface and consists of fibroblast-like cells. The proliferative layer, which is placed under the fibrous layer, serves to separate the fibrocartilaginous fibrous zone from the hypertrophic zones, and has a role as a cell reservoir. Under the proliferative layer, there is a hypertrophic zone, a calcified zone, and a subchondral bone zone. These tissues get nourishment from intra-articular synovial fluid and subchondral bone marrow $[5,6]$. The histological structure of articular eminence is similar to that of condyle. From the outermost layer, there is fibrous articular tissue, proliferative layer, chondrocyte in fibrous matrix, and bone of articular eminence layer [7].

In the structure analysis of biological molecules, chondrocytes are surrounded by a large amount of matrix macromolecules. The macromolecules are composed of proteoglycans, glycosaminoglycans (GAGs), and collagens of type I, II, IX, X, and XI. Hyaluronic acid (HA), a principal GAG, mainly maintains viscosity and causes a decrease in bio rheological properties. Proteoglycans are proteins which constitute the superficial zone of the articular catilage. These proteoglycans include lubricin and chondroitin sulfate substitution. Lubricin is a mucinous glycoprotein with a molecular weight of 345 kilodaltons $(\mathrm{kDa})$ and contains a small amount of keratan. Chondroitin sulfate substitution is present in the superficial layer of articular cartilage and has a role of a boundary lubrication. The collagen fibers of fibrocartilage have tensile and shear strength. Type I collagen mainly presents on the surface of mandibular condyle, and type II and X collagen mainly present in hypertrophic zones which have characteristics of hyaline cartilage [8].

In cartilage which has the configuration explained above, anabolism occurs during dynamic loading, and catabolism occurs during excessive static loading. The resistance to compression depends on the density of proteoglycans. Besides, since most fibers of condylar cartilage travel forward and backward, the cartilage has good resistance to shearing stress, but poor resistance to viscous properties. In addition, when excessive shear stress occurs, the molecular weight of HA decreases and joint lubrication is destroyed $[8,9]$.

\section{PATHOPHYSIOLOGIC MECHANISM OF TEMPOROMANDIBULAR JOINT ARTHRITIS}

\section{Various Factors and Associated Mechanisms of Temporomandibular Joint Arthritis}

TMJ OA was believed to be a cartilage disease, but is now considered as a multi-factorial disease of cartilage, bone (bone marrow), joint disc, ligament, muscle, and nerve [10].

\section{1) Age}

Calcium content of the articular disc increases with age. As the concentration of 6-sulfate disaccharide in chondroitin sulfate increases, the concentration of keratan sulfate, one of GAG, in human cartilage also increases with age. In addition, as the content of GAGs increases, the osmotic swelling pressure of cartilage increases, resulting in cartilaginous stiffness [11,12].

\section{2) Mechanical overload}

Mechanical overload is the main causative factor in the destruction of the mandibular condylar cartilage as in other synovial cartilages. Chondrocytes, especially hypertrophic chondrocytes, have evolved mechanoresponsive mechanisms, which, when activated, increase metabolism, initiate pathological mechanisms, and result in irreversible cartilage destruction $[8,13]$.

At the molecular level, several degeneration processes of articular cartilage due to the recognition of mechanical overload occur. One of them is proteolysis of extracellular matrix components by the plasminogen activator system, and another one is chondrocyte apoptosis by endoplasmic reticulum stress-induced cell death. These processes result in thinning of the condylar cartilage [14]. The initial degenerative changes of osteoarthritis are known to be caused by the downregulation of Indian hedgehog signaling [15]. 
When mechanoresponsive mechanisms are activated, chondrocytes reproduce and proliferate to form various cells. In this reaction, type II collagen, a principal matrix molecule, is synthesized. The splice-variant form of type II collagen is normally expressed during the development of chondroprogenitor cells, however it is also observed in both early and late osteoarthritis. This indicates a potential reversion of chondrocytes to an early developmental phenotype, which may be developed during the degeneration of caltilage [16]. In the case catabolic process is highly activated, many aggrecan fragments degraded by matrix metalloproteinase (MMP) are secreated in synovial fluid. The secreated aggrecan fragments can be used as a marker for increased turnover $[17,18]$.

\section{3) Apoptosis and autophagy of chondrocytes}

Apoptosis of chondrocytes is prominent in the early stages of cartilage degeneration [19]. Oxidative stress formed by an increase in intracellular reactive oxygen species causes chondrocyte apoptosis. Cytokines, developed in the process of chondrocytes apoptosis, cause destruction of subchondral bone. Furthermore, autophagy, normal adaptation process under nutrient stressing conditions, is observed in early articular cartilage degeneration of TMJ OA. The Excess of autophagy can also cause autophagic cell death in chondrocytes. More precisely, rapamycin (a potent autophagy inducer) could protect young chondrocytes, whereas excessive activation of rapamycin results in autophagic cell death in osteoarthritis chondrocytes [19,20].

\section{4) Catabolic enzymes}

Activation of various catabolic enzymes such as MMP, aggrecanases, disintegrin, and metalloproteinase with thrombospondin motifs is involved in the TMJ OA. Nuclear factor (NF)- $\kappa B$ signaling pathway induces upregulation of Wnt-5A, and as a result of this process, development of interleukin (IL)-1 $\beta$-induced catabolism, and elevated expression of high-temperature requirement serine protease A1 (HtrA1) is observed in the early stages of TMJ OA, and elevated expression of HtrA1 initiates the degradation of the chondrocyte pericellular matrix, especially type II collagen. The major mediators of cartilage degeneration are MMP-1, $-3,-9$, and -13 that metabolize aggrecan, and collagen type
II, and aggrecanases (aggrecanase-1 and -2) that metabolize aggrecan [21,22].

\section{5) Inflammation}

In synovial tissues and synovial fluid with TMJ OA, inflammatory mediators such as IL-12, IL-1 $\beta$, IL-6, IL-17, IL23 , tumor necrosis factor- $\alpha$, and monocyte chemoattractant protein (MCP)-1 are observed, among which MCP-1 plays an important role in recruiting the mononuclear cells in synovial fluid. These changes cause a decrease of the lubrication in the synovial fluid and a degeneration of the subchondral bone [23,24].

\section{6) Abnormal remodeling of subchondral bone}

Chondrocytes contribute to the destruction of subchondral bone by regulating the receptor activator of NF- $\mathrm{NB}$ ligand and the osteoprotegerin (OPG). Stromal cells-derived factor 1, MMP-9, and IL-6, affected by chondrocytes, contribute to subchondral bone remodeling $[25,26]$.

Transforming growth factor beta 1 acts as an initiator of subchondral bone turnover increase and bone density decrease in TMJ OA, and causes an increase in apoptosis by upregulation of MMP-9, MMP-13, and vascular endothelial growth factor in mandibular condyle chondrocytes [27].

\section{7) Estrogen}

17b-estradiol induces the secretion of OPG and protects bones from systemic and local inflammatory factors. When it is deficient, inflammation is caused by various factors, subsequently bone resorption is developed, and new bone formations are inhibited [28].

Mandibular condyle proliferation is inhibited by an estrogen receptor (ER)- $\beta$-dependent mechanism. Subchondral bone and cartilage degeneration driven by upregulation of Fas and caspase 3-related proapoptotic genes is inhibited by ER antagonists. In contrast, estrogen has a protective effect on TMJ chondrocytes by inhibiting the expression of nitric oxide [29]. Therefore, the function of estrogen in the pathogenesis of TMJ OA has not been clearly identified. In addition, the effects of other female hormones, including relaxins and progestins, on the progression of cartilage degradation in TMJ OA should be further evaluated [14]. 


\section{Association of Various Tissues and Organs for Osteoarthritis}

1) Muscle

Traditionally, pathologic conditions of muscle have been considered to occur secondary to joint pain, but recently, it is believed that abnormal muscle movements and sensory dysfunctions of muscle may contribute to the cause of arthritis. In addition, changes in the muscles were observed before the onset of joint pain. However, the exact mechanism has not yet been elucidated [30,31].

2) Obesity and adipose tissue

Adipose tissue is an active endocrine organ and secretes adipokines, leptin, adiponectin, and resistin. Leptin affects osteoblasts and chondrocytes, and adiponectin plays a proinflammatory action. Bone marrow contains arachidonic acid, which contributes to the production of prostaglandins. The association of osteoarthritis histological severity and lipid accumulation in cartilage was reported [32-34]. In a TMJ OA study, the degeneration of the joint was associated with mechanical overload and high-fat diet [35].

\section{3) Nervous system}

The nervous system, the endocrine system, and the musculoskeletal system influence one another. Leptin affects bone through the hypothalamic pathway, and severe sensory neuropathy causes rapid joint destruction [36]. Various neuropeptides and enzymes, such as substance P, alphacalcitonin gene-related peptide, vasointestinal peptide $\mathrm{P}$, pituitary adenylate cyclase-activating peptide, neuropeptide Y, serotonin, glutamate, tyrosine hydroxylase and norepinephrine have related receptors in bone cell lineages [37]. Cocaine and amphetamine-regulated transcript, melanocortin 4 receptor and the cannabinoid receptor CB1 regulate bone remodeling and energy homeostasis, all of which show high levels of expression in the hypothalamus [38].

\section{4) Vascular changes}

Vascular infiltration from the subchondral bone to the calcified cartilage is observed in early arthritis [39]. These microvascular changes may change the subchondral bone blood flow, which consequentially changes the nutrition of the cartilage, or cause bone ischemia to accelerate the progression of arthritis $[40,41]$.

\section{CONCLUSION}

TMJ OA is a low-level inflammatory disease characterized by degeneration of articular cartilage and subchondral bone. The TMJ OA is induced by dysregulation of the catabolic and anabolic activity of chondrocytes. The main cause of the dysregulation is mechanical overload, and other associated factors are age and estrogen. Muscle dysfuction, adipose tissue, nervous system, and vascular changes may be involved.

TMJ OA should be diagnosed under consideration of its multifactorial disease characteristics. Many studies are trying to make an accurate diagnosis based on various factors reflecting the pathophysiological mechanism, radiographic and clinical findings of TMJ OA [42-44], and further efforts should be made for early and accurate diagnosis and management of TMJ OA through the development of diagnostic markers which reflect various mechanisms.

\section{CONFLICT OF INTEREST}

No potential conflicts of interest relevant to this article are reported.

\section{ORCID}

\author{
Hye-Min Ju \\ https://orcid.org/0000-0002-9252-6717 \\ Kyung-Hee Kim \\ https://orcid.org/0000-0003-2922-6452 \\ Sung-Hee Jeong \\ https://orcid.org/0000-0002-6296-4775 \\ Yong-Woo Ahn \\ https://orcid.org/0000-0002-2197-0394 \\ Soo-Min Ok \\ https://orcid.org/0000-0003-1776-371X
}

\section{REFERENCES}

1. Yadav S, Yang Y, Dutra EH, Robinson JL, Wadhwa S. Temporomandibular joint disorders in older adults. J Am Geriatr Soc 
2018;66:1213-1217.

2. Schiffman E, Ohrbach R, Truelove E, et al.; International RDC/ TMD Consortium Network, International association for Dental Research; Orofacial Pain Special Interest Group; International Association for the Study of Pain. Diagnostic criteria for temporomandibular disorders (DC/TMD) for clinical and research applications: recommendations of the International RDC/TMD Consortium Network and Orofacial Pain Special Interest Group. J Oral Facial Pain Headache 2014;28:6-27.

3. Chen D, Shen J, Zhao W, et al. Osteoarthritis: toward a comprehensive understanding of pathological mechanism. Bone Res 2017;5:16044.

4. Kalladka M, Quek S, Heir G, Eliav E, Mupparapu M, Viswanath A. Temporomandibular joint osteoarthritis: diagnosis and long-term conservative management: a topic review. J Indian Prosthodont Soc 2014;14:6-15.

5. Wang Y, Wei L, Zeng L, He D, Wei X. Nutrition and degeneration of articular cartilage. Knee Surg Sports Traumatol Arthrosc 2013;21:1751-1762.

6. Lv X, Li Q, Wu S, Sun J, Zhang M, Chen YJ. Psychological stress alters the ultrastructure and increases IL- $1 \beta$ and TNF- $\alpha$ in mandibular condylar cartilage. Braz J Med Biol Res 2012;45:968-976.

7. Hinton RJ, Carlson DS. Histological changes in the articular eminence and mandibular fossa during growth of the rhesus monkey (Macaca mulatta). Am J Anat 1983;166:99-116.

8. Kuroda S, Tanimoto K, Izawa T, Fujihara S, Koolstra JH, Tanaka E. Biomechanical and biochemical characteristics of the mandibular condylar cartilage. Osteoarthritis Cartilage 2009;17:1408-1415.

9. Rath B, Nam J, Knobloch TJ, Lannutti JJ, Agarwal S. Compressive forces induce osteogenic gene expression in calvarial osteoblasts. J Biomech 2008;41:1095-1103.

10. Man GS, Mologhianu G. Osteoarthritis pathogenesis - a complex process that involves the entire joint. J Med Life 2014;7:37-41.

11. Nakano T, Scott PG. Changes in the chemical composition of the bovine temporomandibular joint disc with age. Arch Oral Biol 1996;41:845-853.

12. Crockett AB, Wiersma GB, Tai H, Mitchell W. Pesticide and mercury residues in commercially grown catfish. Pestic Monit J 1975;8:235-240.

13. Nordberg RC, Mellor LF, Krause AR, Donahue HJ, Loboa EG. LRP receptors in chondrocytes are modulated by simulated microgravity and cyclic hydrostatic pressure. PLoS One 2019;14:e223245.

14. Lee YH, Park HK, Auh QS, et al. Emerging potential of exosomes in regenerative medicine for temporomandibular joint osteoarthritis. Int J Mol Sci 2020;21:1541.

15. Zhou J, Chen Q, Lanske B, et al. Disrupting the Indian hedgehog signaling pathway in vivo attenuates surgically induced osteoarthritis progression in Col2a1-CreERT2; Ihhfl/fl mice. Arthritis Res Ther 2014;16:R11.

16. Aigner T, Zhu Y, Chansky HH, Matsen FA 3rd, Maloney WJ, Sandell LJ. Reexpression of type IIA procollagen by adult articular chondrocytes in osteoarthritic cartilage. Arthritis Rheum 1999;42:1443-1450.

17. Goldring MB, Goldring SR. Osteoarthritis. J Cell Physiol 2007;213:626-634.
18. Lohmander LS, Ionescu M, Jugessur H, Poole AR. Changes in joint cartilage aggrecan after knee injury and in osteoarthritis. Arthritis Rheum 1999;42:534-544.

19. Yang H, Wen Y, Zhang M, et al. MTORC1 coordinates the autophagy and apoptosis signaling in articular chondrocytes in osteoarthritic temporomandibular joint. Autophagy 2020;16:271288.

20. Chang J, Wang W, Zhang H, Hu Y, Wang M, Yin Z. The dual role of autophagy in chondrocyte responses in the pathogenesis of articular cartilage degeneration in osteoarthritis. Int J Mol Med 2013;32:1311-1318.

21. Yamashita-Futani Y, Jokaji R, Ooi K, et al. Metalloelastase-12 is involved in the temporomandibular joint inflammatory response as well as cartilage degradation by aggrecanases in STR/Ort mice. Biomed Rep 2021;14:51.

22. Wong M, Siegrist M, Goodwin K. Cyclic tensile strain and cyclic hydrostatic pressure differentially regulate expression of hypertrophic markers in primary chondrocytes. Bone 2003;33:685-693.

23. Zwiri A, Al-Hatamleh MAI, W Ahmad WMA, et al. Biomarkers for temporomandibular disorders: current status and future directions. Diagnostics (Basel) 2020;10:303.

24. Matsumoto K, Honda K, Ohshima M, et al. Cytokine profile in synovial fluid from patients with internal derangement of the temporomandibular joint: a preliminary study. Dentomaxillofac Radiol 2006;35:432-441.

25. Zhou X, Cao H, Yuan Y, Wu W. Biochemical signals mediate the crosstalk between cartilage and bone in osteoarthritis. Biomed Res Int 2020;2020:5720360.

26. Chien SY, Tsai CH, Liu SC, et al. Noggin inhibits IL-1 $\beta$ and BMP2 expression, and attenuates cartilage degeneration and subchondral bone destruction in experimental osteoarthritis. Cells 2020;9:927.

27. Wang XD, Zhang JN, Gan YH, Zhou YH. Current understanding of pathogenesis and treatment of TMJ osteoarthritis. J Dent Res 2015;94:666-673.

28. Gunson MJ, Arnett GW, Formby B, Falzone C, Mathur R, Alexander C. Oral contraceptive pill use and abnormal menstrual cycles in women with severe condylar resorption: a case for low serum 17beta-estradiol as a major factor in progressive condylar resorption. Am J Orthod Dentofacial Orthop 2009;136:772-779.

29. Wang XD, Kou XX, Meng Z, et al. Estrogen aggravates iodoacetate-induced temporomandibular joint osteoarthritis. J Dent Res 2013;92:918-924.

30. Kawai N, Tanaka E, Langenbach GE, et al. Jaw-muscle activity changes after the induction of osteoarthrosis in the temporomandibular joint by mechanical loading. J Orofac Pain 2008;22:153162.

31. Brandt KD, Heilman DK, Slemenda C, et al. Quadriceps strength in women with radiographically progressive osteoarthritis of the knee and those with stable radiographic changes. J Rheumatol 1999;26:2431-2437.

32. Cornish J, Callon KE, Bava U, et al. Leptin directly regulates bone cell function in vitro and reduces bone fragility in vivo. J Endocrinol 2002;175:405-415.

33. Ehling A, Schäffler A, Herfarth H, et al. The potential of adipo- 
nectin in driving arthritis. J Immunol 2006;176:4468-4478.

34. Lippiello L, Walsh T, Fienhold M. The association of lipid abnormalities with tissue pathology in human osteoarthritic articular cartilage. Metabolism 1991;40:571-576.

35. Du J, Jiang Q, Mei L, et al. Effect of high fat diet and excessive compressive mechanical force on pathologic changes of temporomandibular joint. Sci Rep 2020;10:17457.

36. Ducy P, Amling M, Takeda S, et al. Leptin inhibits bone formation through a hypothalamic relay: a central control of bone mass. Cell 2000;100:197-207.

37. Bjurholm A. Neuroendocrine peptides in bone. Int Orthop 1991;15:325-329.

38. Rosen CJ. Bone remodeling, energy metabolism, and the molecular clock. Cell Metab 2008;7:7-10.

39. Suri S, Gill SE, Massena de Camin S, Wilson D, McWilliams DF, Walsh DA. Neurovascular invasion at the osteochondral junction and in osteophytes in osteoarthritis. Ann Rheum Dis
2007;66:1423-1428.

40. Findlay DM. Vascular pathology and osteoarthritis. Rheumatology (Oxford) 2007;46:1763-1768.

41. Sharma AR, Jagga S, Lee SS, Nam JS. Interplay between cartilage and subchondral bone contributing to pathogenesis of osteoarthritis. Int J Mol Sci 2013;14:19805-19830.

42. Wang X, Yu Y, Huang Y, et al. Identification of potential diagnostic gene biomarkers in patients with osteoarthritis. Sci Rep 2020;10:13591.

43. Ok SM, Lee SM, Park HR, Jeong SH, Ko CC, Kim YI. Concentrations of CTX I, CTX II, DPD, and PYD in the urine as a biomarker for the diagnosis of temporomandibular joint osteoarthritis: a preliminary study. Cranio 2018;36:366-372.

44. Bianchi J, de Oliveira Ruellas AC, Gonçalves JR, et al. Osteoarthritis of the Temporomandibular Joint can be diagnosed earlier using biomarkers and machine learning. Sci Rep 2020;10:8012. 